\title{
Relación entre perímetro abdominal, nivel socioeconómico y presión arterial
}

\author{
Eduardo Fasce H, Fabrizio Fasce V, Hernán Zárate M, Ivone Campos C ${ }^{\mathrm{a}}$, Maritza Flores $\mathrm{O}^{\mathrm{b}}$ \\ y Pilar lbáñez Gc. \\ ${ }^{a}$ Enfermera \\ ${ }^{b}$ Bioestadística \\ c Matrona \\ Facultad de Medicina, Universidad de Concepción, Departamentos de Educación Médica y \\ Salud Pública. \\ Recibido el 16 de Noviembre de 2009, Aceptado el 25 de Febrero del 2010
}

Rev Chil Cardiol 2010; 29: 11-18

\section{Resumen:}

Objetivos: Relacionar perímetro abdominal y condición socioeconómica con presión arterial (PA) en comunidad urbana de Concepción.

Métodos: Se midió el perímetro abdominal $(P e A)$ de 8472 residentes mayores de 15 años de edad, proporcionados por edad, género y nivel socioeconómico. La PA fue medida con normas estandarizadas, realizando dos visitas en diferentes días, la segunda si la PA era mayor de 140/90 mm Hg. Los PeA se distribuyeron en cuartiles, relacionando cada cuartil con los respectivos promedios de presión sistólica y diastólica y se establecieron coeficientes de correlación lineal mediante " $r$ " de Pearson entre PeA y PA. Además, se estableció la frecuencia de cada cuartil de PeA por nivel socioeconómico.

Resultados: Los Pea tuvieron la siguiente distribución (cm): 1er cuartil, < 78; $2^{\circ}$ cuartil, 78 - 87; 3er cuartil, 88 - 96 y $4^{\circ}$ cuartil, > 96. La prevalencia de hipertensión para niveles socioeconómicos alto, medio y bajo fue 17,9\%, 19,5\% y $24,5 \%$, respectivamente. La PA promedio $(\mathrm{mmHg})$ en hombres y en mujeres del 1er cuartil de PeA fue 112 y 109 ; en el $2^{\circ}$ cuartil 118 y 118; en el 3er cuartil 123 y 122 y en el $4^{\circ}$ cuartil 129 y 129, respectivamente. Los coeficientes de correlación entre PeA y presión sistólica y presión diastólica resultaron significativos en ambos géneros: $r=0,343$, $p<0.00001$ y $r=0,281, p<0.00001$, respectivamente. La distribución (\%) de PeA fue inversa al nivel socioeconómico: en el nivel social bajo, el $22 \%$ estaba en el 1 er cuartil de $P$ eA, y el $28,8 \%$ en el $4^{\circ}$ cuartil. En el nivel social alto se observó lo inverso: $38,9 \%$ estaba en el 1er cuartil de PeA, y sólo el $15.5 \%$ en el $4^{\circ}$ cuartil $(p<0.0001)$.

Conclusiones: Hubo correlación positiva entre PeA y PA en ambos géneros. La frecuencia de mayor PeA en el nivel socioeconómico bajo podría explicar la mayor prevalencia de hipertensión en este grupo.

Correspondencia: Eduardo Fasce $\mathrm{H}$.

Facultad de Medicina, Universidad de Concepción,

Barrio Universitario s/n.

Concepción, Región del Bío Bío, Chile

Fax: (56) (41) 2215478

Correo Electrónico: efasce@udec.cl 


\title{
Relation of abdominal circumference and socio-economic status to blood pressure
}

\begin{abstract}
Aim: To correlate abdominal circumference (AC) and socio-economic status with blood pressure( $B P)$ in an urban community of Concepción, Chile

Methods: AC was measured in 8472 subjects above 15 years of age, stratified by age, gender and socio-economic status. BP was measured by standard procedures, with a repeat recording when the initial value was $>140 / 90 \mathrm{mmHg}$. $B P$ was compared in quartiles of abdominal circumference and according to socio-economic status. Pearson " $r$ " was used to correlate $B P$ and $A C$
\end{abstract}

Results: Cut points for quartiles of $A C$ were 78,87 , and $96 \mathrm{~cm}$. Prevalence of hypertension in high, medium and low socio-economic status was 17.9\%, 19.5\% and 24.5\%, respectively. Mean systolic BP was 112 - $109 \mathrm{mmHg}$ (males - females) in the first AC quartile, 118 - 118, 123-122 and 129-129 in the second, third and fourth quartiles, respectively. A significant correlation between AC and BP (systolic and diastolic) was observed in both genders ( $r$ 0.345 and 0.281 for males and females, respectively, $p<0.00001) .22 \%$ of low socio-economic subjects belonged in the first AC quartile compared to $28.8 \%$ in the 4 th quartile. In contrast $38.9 \%$ of high socio-economic subjects belonged in the first AC quartile while $15.5 \%$ did so in the 4 th quartile $(p<0001)$.

Conclusion: A positive correlation of AC and blood pressure was shown in both genders. A greater AC in low socioeconomic subjects may be related to a higher prevalence of hypertension in this group.

Key words: abdominal circumference, socio-economic status, blood pressure

\section{Introducción}

El sobrepeso y la obesidad han experimentado un progresivo aumento en las últimas décadas ${ }^{1}$. De acuerdo al estudio Framingham, en el transcurso de los últimos 50 años su respectiva incidencia se ha duplicado y triplicado ${ }^{2}$. A nivel mundial, tanto en países desarrollados como en vías de desarrollo, se estima que la prevalencia varía entre $40 \%$ y $60 \%{ }^{3}$. En Chile, de acuerdo a la Encuesta Nacional de Salud del año 2003, la prevalencia de sobrepeso fue de $37,8 \%$ y de $23,3 \%$ para la obesidad, incluida la obesidad mórbida ${ }^{4}$.

En diferentes estudios epidemiológicos, entre otros Framingham y National Health and Nutritional Examination Surveys (NHANES), se ha reconocido una estrecha correlación entre peso corporal y presión arterial, pudiendo estimarse que alrededor del $70 \%$ de la hipertensión sería atribuible al exceso de adiposidad 5 . Por otra parte, en el estudio INTERHEART, destinado a identificar los factores de riesgo de infarto del miocardio, la obesidad abdominal ocupó el nivel más alto de riesgo poblacional atribuible ${ }^{6}$. La importancia de la relación entre obesidad e hipertensión, y ambas, con el riesgo cardiovascular, determinó la elaboración de un documento de trabajo especial de la Sociedad Europea de Hipertensión, dirigido a recopilar los antecedentes disponibles y establecer metas y perspectivas ${ }^{7}$.

De igual modo, la prevalencia de hipertensión arterial en las comunidades urbanas de Concepción, Chiguayante y San Pedro de la Paz, ha experimentado un incremento de $17 \%$ en un período de 15 años, desde $18,6 \%$ en 1988 a $21,7 \%$ en $2004^{8}$. Este comportamiento de la hipertensión es concordante con los resultados obtenidos por Patricia Kearney a través de un análisis de la literatura mundial, estimando un aumento desde $26,4 \%$ correspondiente al año 2000 , al $29,2 \%$ proyectado para el año $2025^{9}$.

En consideración a estos antecedentes nos propusimos establecer la prevalencia de hipertensión de acuerdo al nivel socioeconómico de la población urbana de Concepción, Chiguayante y San Pedro de la Paz, y relacionar los perímetros de cintura con la presión arterial y el nivel socioeconómico. 


\section{Material y Métodos}

La metodología utilizada en este estudio se encuentra detallada en una comunicación anterior ${ }^{8}$.

En resumen, correspondió a un estudio de campo realizado en las comunas de Concepción, Chiguayante y San Pedro de la Paz, en una muestra no institucional de 8.472 residentes permanentes, mayores de 15 años, proporcionados poredad, géneroy nivel socioeconómico. Este último criterio se basó en los datos de la Encuesta de Caracterización Socioeconómica Nacional (Casen) $2003^{10}$.

La presión arterial fue medida por personal debidamente entrenado utilizando esfigmomanómetros mercuriales de acuerdo a normas estandarizadas ${ }^{11}$. Se realizaron dos controles de presión en una primera visita y un segundo control en un día posterior cuando la presión arterial era igual o mayor a $140 / 90 \mathrm{~mm} \mathrm{Hg}$ en sujetos sin terapia. Se consideró como hipertenso a todo sujeto con presión sistólica $\geq 140 \mathrm{~mm} \mathrm{Hg}$ y/o presión diastólica $\geq 90 \mathrm{~mm} \mathrm{Hg}$ determinada en el último registro, y sujetos en tratamiento independientemente de su nivel de presión.

El perímetro abdominal fue medido mediante huinchas graduadas, con el sujeto en posición de pie, con la piel del abdomen descubierta, en un punto intermedio entre la última costilla y la cresta ilíaca ${ }^{12}$.

Los perímetros abdominales se agruparon en cuartiles los cuales fueron relacionados con los niveles de presión arterial mediante la determinación de correlación lineal "r" de Pearson.

Se estableció la prevalencia de hipertensión para cada nivel socioeconómico, alto, medio y bajo, estando éstos agrupados de acuerdo a la información de encuesta CASEN.

\section{Resultados}

La separación de perímetros abdominales en cuartiles tuvo la siguiente distribución en $\mathrm{cm}$ : 1 er cuartil, $<78 ; 2^{\circ}$ cuartil, 78 - 87; 3er cuartil, $88-96$ y $4^{\circ}$ cuartil, $>96$.

La distribución porcentual de cuartiles de perímetro abdominal por nivel socioeconómico se detalla en la tabla 1.

Tabla 1. Distribución (\%) de cuartiles de perímetro abdominal en los niveles socioeconómicos alto, medio y bajo

NIVEL SOCIOECONÓMICO

\begin{tabular}{lccc}
\hline CUARTILES $(\mathrm{cm})$ & ALTO & MEDIO & BAJO \\
$1^{\circ}(<78)$ & 38,9 & 21 & 22 \\
$2^{\circ}(78-87)$ & 26,8 & 26,9 & 24,7 \\
$3^{\circ}(88-96)$ & 18,8 & 27,3 & 24,4 \\
$4^{\circ}(>96)$ & 15,5 & 24,9 & 28,9 \\
TOTAL & 100 & 100 & 100 \\
\hline
\end{tabular}

Las diferencias entre $1 \mathrm{er}$ y $4^{\circ}$ cuartil de perímetro abdominal correspondiente a los niveles socioeconómicos alto y bajo, obtenidas mediante $\mathrm{chi}^{2}$ de Pearson, fueron altamente significativas $(p<0.0001$, gráfico 1).

Las tasas de prevalencia de hipertensión para los niveles socioeconómicos alto, medio y bajo fueron de $17,9 \%, 19,5 \%$ y $24,5 \%$, respectivamente (gráfico
$2)$, diferencias que resultaron altamente significativas $(p<0.0001)$.

Los promedios de presión sistólica y de presión diastólica aumentaron significativamente en ambos géneros en relación al aumento del perímetro abdominal expresado en cuartiles (gráficos 3 y 4 , respectivamente).

Estas diferencias ocurrieron en todos los tramos de 
E. Fasce, F. Fasce, H. Zárate, I. Campos, M. Flores, P. Ibáñez.

edad en ambos géneros (Tabla 2).

Los coeficientes de correlación lineal entre presión sistólica y presión diastólica y perímetro abdominal, obtenidos mediante "r" de Pearson, fueron altamente significativos $(r=0,343, p<0.00001$, para presión sistólica y $r=0,281, p<0.00001$, para presión diastólica, gráfico 5).

\section{Discusión}

ALTO $\square$ BAJO

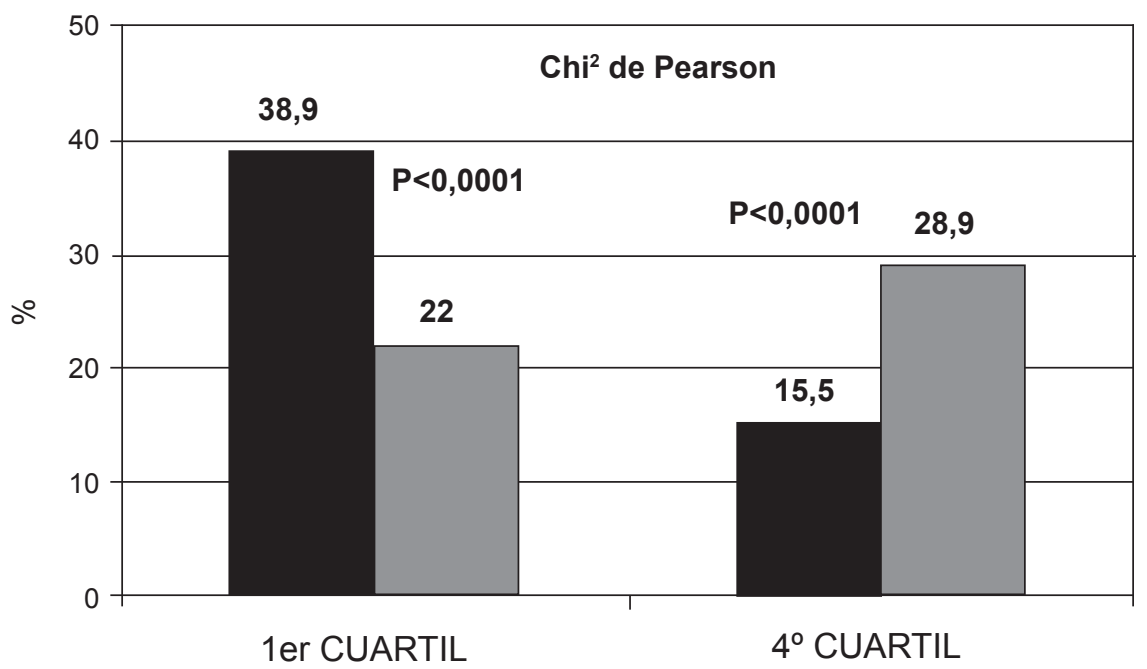

Gráfico1: Comparación de frecuencia (\%) de 1er y $4^{\circ}$ cuartil de perímetro abdominal entre niveles socioeconómicos alto y bajo.

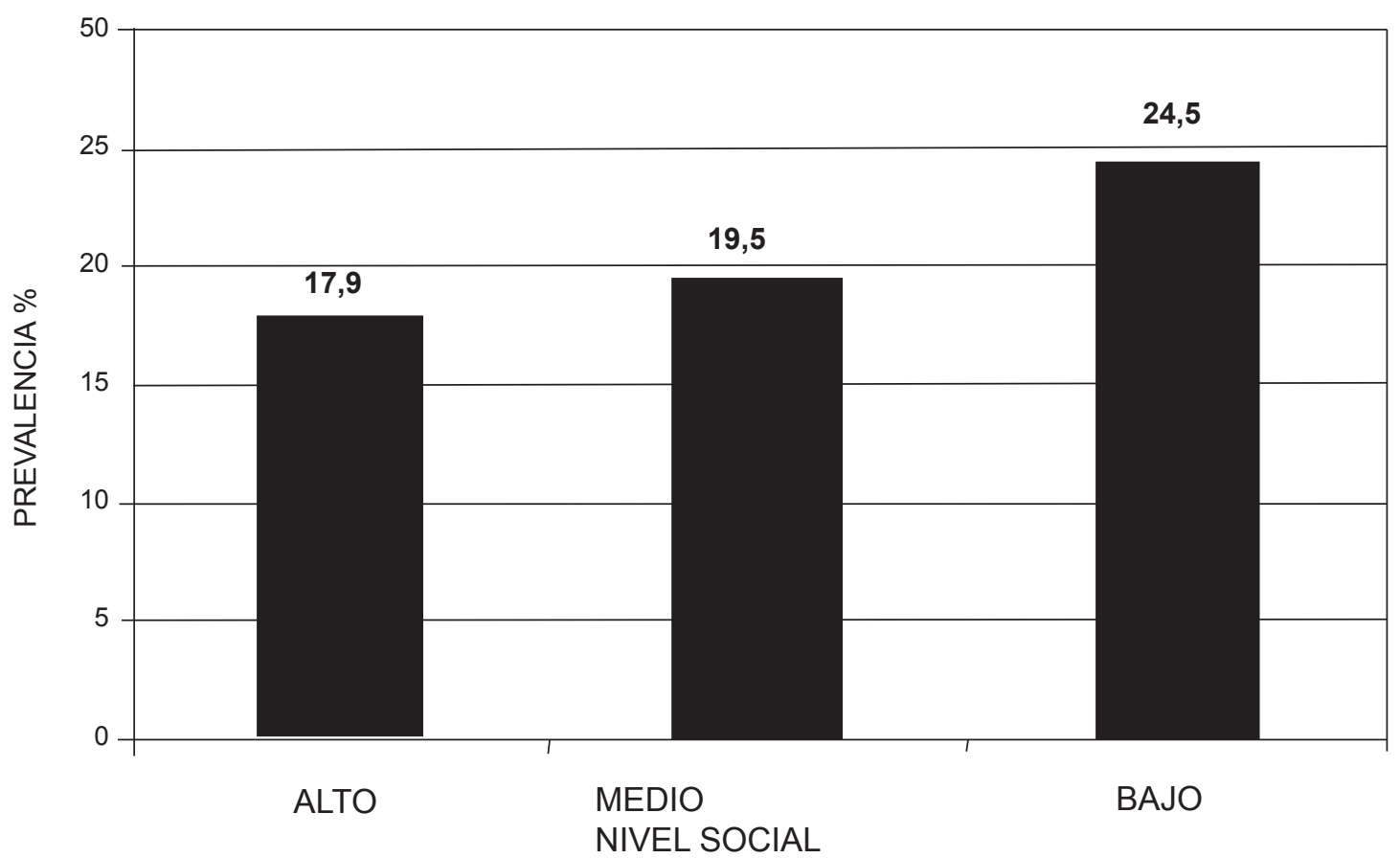

Gráfico 2: Prevalencia de hipertensión arterial (\%) según nivel socioeconómico alto, medio y bajo 


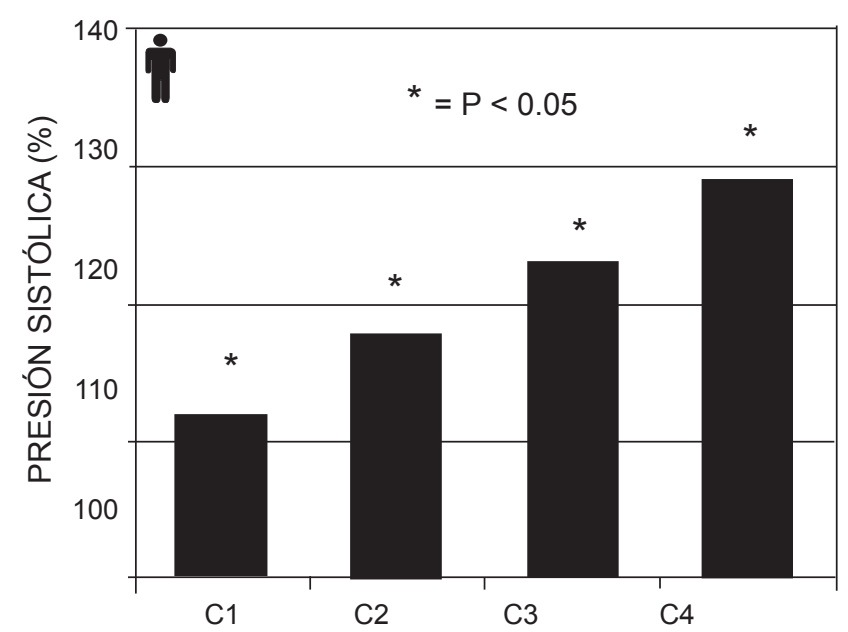

PERIMETROS DE CINTURA EN CUARTILES

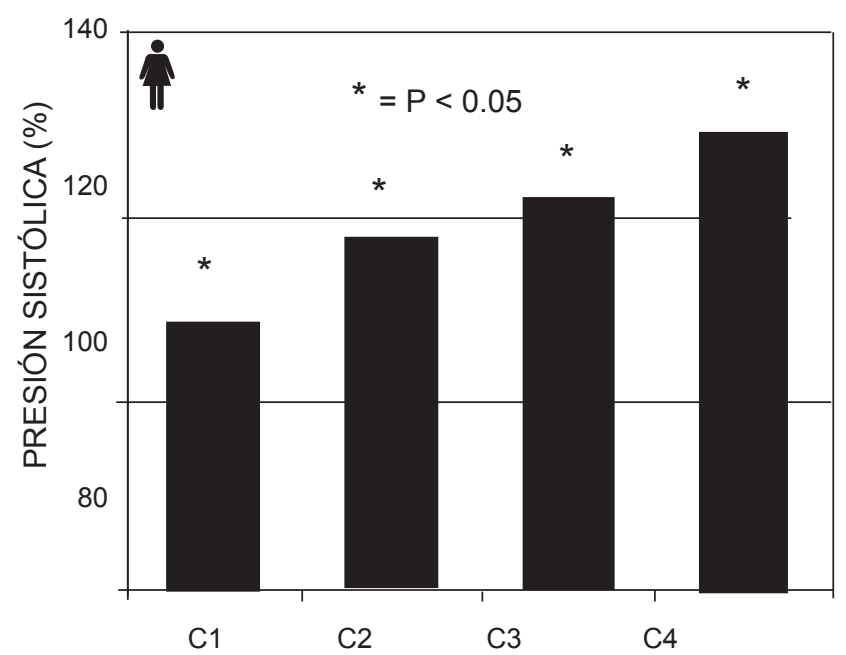

PERIMETROS DE CINTURA EN CUARTILES

Gráfico 3: Promedio de presión sistólica en hombres (a izquierda) y mujeres (derecha) según cuartiles de perímetro abdominal

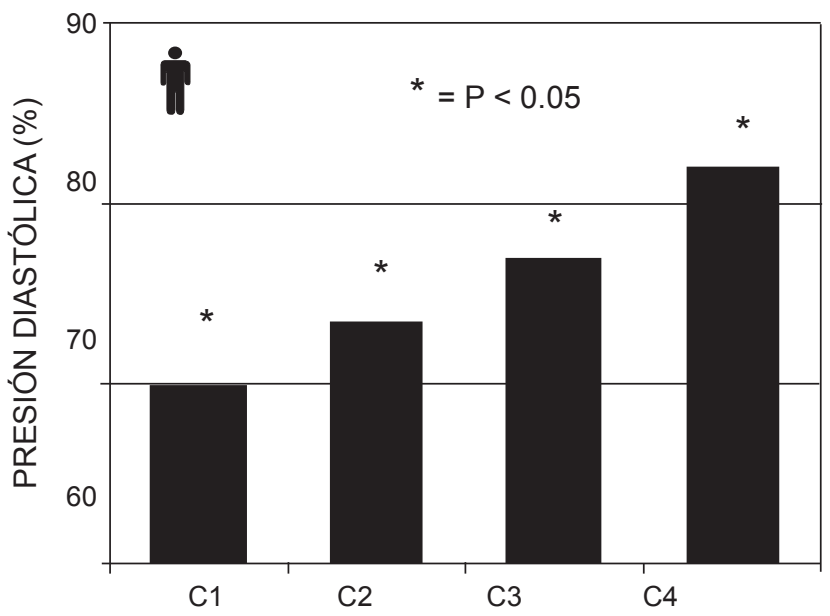

PERIMETROS DE CINTURA EN CUARTILES

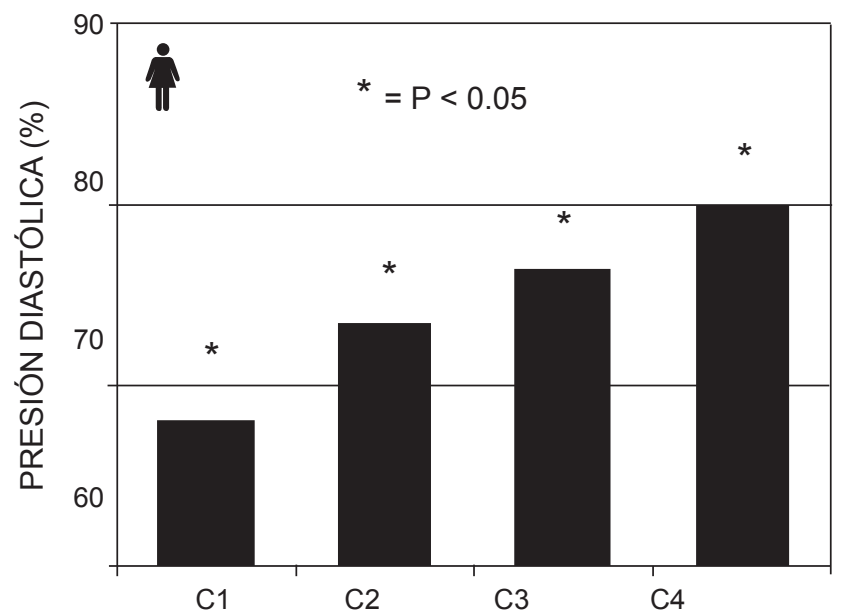

PERIMETROS DE CINTURA EN CUARTILES

Gráfico 4: Promedio de presión diastólica en hombres (a izquierda) y mujeres (derecha) según cuartiles de perímetro abdominal

Tabla 2. Promedios de presión arterial sistólica por edad y género según cuartiles de perímetro abdominal

\begin{tabular}{lllllllll}
\hline & \multicolumn{3}{c}{ MASCULINO } & \multicolumn{5}{c}{ FEMENINO } \\
\hline EDAD & C1 & C2 & C3 & C4 & C1 & C2 & C3 & C4 \\
\hline $15-24$ & 111 & 114 & 116 & 120 & 105 & 107 & 111 & 116 \\
$25-34$ & 113 & 117 & 119 & 122 & 109 & 114 & 115 & 119 \\
$35-44$ & 117 & 118 & 121 & 125 & 112 & 115 & 119 & 125 \\
$45-54$ & 113 & 122 & 123 & 126 & 117 & 122 & 123 & 129 \\
$55-64$ & 118 & 127 & 121 & 136 & 121 & 127 & 130 & 133 \\
$65-74$ & 129 & 132 & 132 & 134 & 128 & 134 & 133 & 139 \\
\hline
\end{tabular}

C1 $=1$ er cuartil; C2 $=2^{\circ}$ cuartil; C3 $=3^{\circ}$ cuartil; C4 $=4^{\circ}$ cuartil 


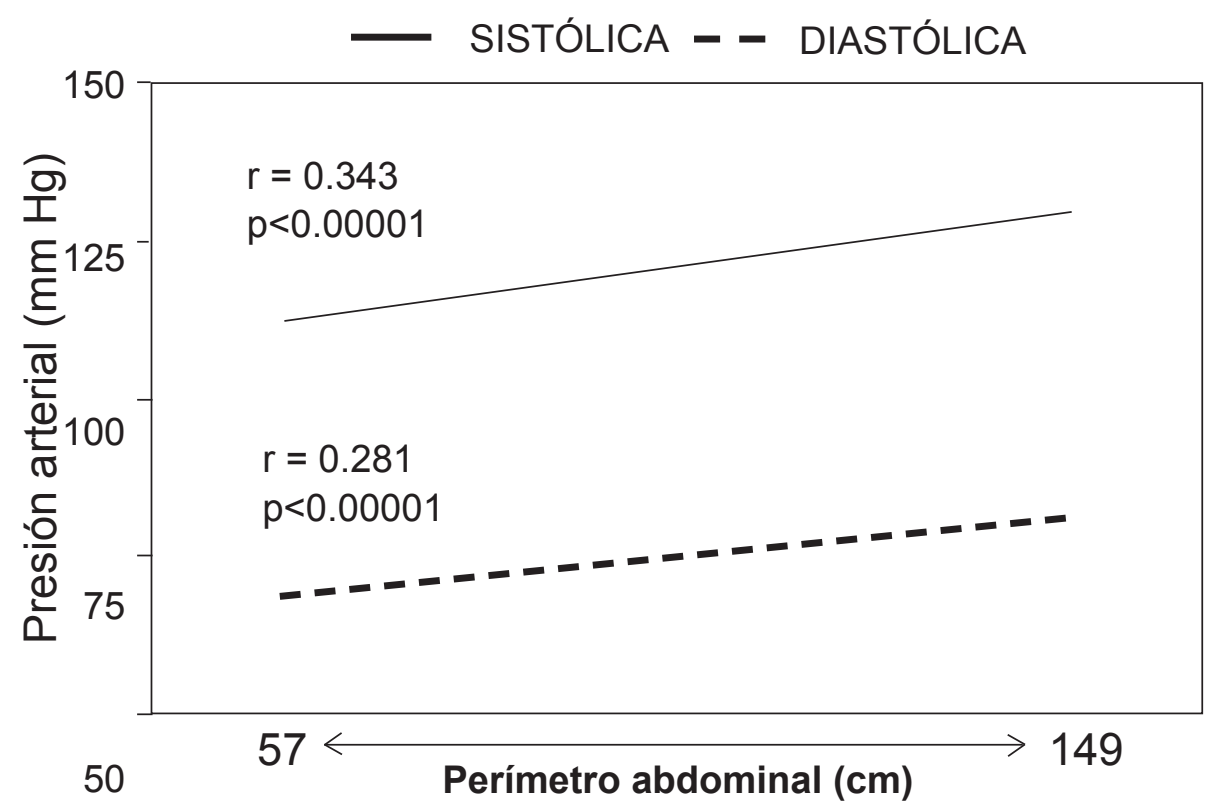

Gráfico 5: Coeficiente de correlación lineal entre perímetro de cintura y presiones sistólica y diastólica

La mayor dificultad para establecer comparaciones entre tasas de prevalencia de hipertensión provenientes de diferentes estudios epidemiológicos es la falta de concordancia en las metodologías utilizadas $^{13,14}$. Las principales diferencias se refieren a los dinteles considerados para separar normotensión de hipertensión, el número de registros efectuados, el número de visitas en que se realizaron las mediciones, el tipo de esfigmomanómetro, la representatividad de la muestra, los límites de edad de la población de estudio, etc ${ }^{15}$. Por otra parte, la comparación de las tasas obtenidas en países o comunidades con diferentes niveles de desarrollo, las que se han estimado como representativas de diferencias en la condición socioeconómica, está influenciada por determinantes genéticos, de raza o etnia, y por el impacto de los diversos factores ambientales y de estilos de vida, siendo estos últimos los que se han comprobado como de mayor importancia ${ }^{16}$.

El diseño metodológico del presente estudio permite garantizar la exclusión de los sesgos antes mencionados. Al considerar una muestra representativa de una misma comunidad, utilizando la clasificación de los niveles socioeconómicos mediante instrumentos válidos (encuesta Casen ${ }^{10}$ ), se otorga objetividad a la comparación de las tasas de prevalencia que, en este caso, resultaron inversas al nivel socioeconómico: $17,9 \%, 19,5 \%$ y $24,5 \%$ para los niveles socioeconómicos alto, medio y bajo, respectivamente. Estos índices son más elevados que los comunicados por Jadue y cols en la población de Valparaíso ${ }^{17}$ en el año 1997, probablemente debido a las diferencias en los rangos de edad entre ambas muestras poblacionales.

Aún cuando la relación entre obesidad y presión arterial ha quedado establecida en numerosos estudios $^{18-30}$, no todo paciente obeso es hipertenso ${ }^{31}$, y la asociación entre obesidad e hipertensión varía entre poblaciones con diferencias étnicas o raciales ${ }^{27,32}$.

Dadas las características de la muestra motivo de esta investigación es posible establecer con mayor precisión la relación entre adiposidad abdominal y nivel socioeconómico, comprobando un aumento significativo de los perímetros abdominales en los estratos socioeconómicos más bajos. La utilización del perímetro de cintura como criterio para determinar 
la adiposidad abdominal no sólo se sustenta en su valor práctico para ser aplicado en estudios epidemiológicos con universos muestrales amplios, sino también por ser considerado un índice clínico de alta confiabilidad para relacionar la adiposidad con la presión arterial ${ }^{21,25}$, siendo la adiposidad abdominal la que se ha demostrado más vinculada a la presión arterial y a la hipertensión ${ }^{18-21,26,27}$ y con el riesgo cardiovascular ${ }^{3}$. Consecuente con lo anterior, Majane y cols. $^{33}$ comprueban que la medición del perímetro abdominal constituye el índice de adiposidad que más se correlaciona con la presión arterial ambulatoria de 24 horas.

A través del presente estudio se ha logrado establecer una correlación inversa entre nivel socioeconómico v/s perímetro abdominal y presión arterial, y una relación directa entre perímetro abdominal y presión arterial.

Dados los diversos mecanismos que vinculan la adiposidad abdominal con la presión arterial y la hipertensión ${ }^{34}$, los que incluyen el aumento de la actividad simpática mediada por leptina, la activación del sistema renina angiotensina, la disfunción endotelial, los cambios renales estructurales y la apnea obstructiva del sueño, cabe postular que la mayor adiposidad abdominal comprobada en los niveles socioeconómicos más bajos pudiese ser responsable, a lo menos en parte, de las tasas de prevalencia de hipertensión más altas detectadas en este grupo.

REFERENCIAS:

1. ALLENDER S, RAYNER M. The burden of overweight and obesity-related ill health in the UK. Obes Rev 2007; 8: 46773.

2. PARIHK N, PENCINA M, WANG T, LANIER K, FOX C, D'AGOSTINO $R$, et al. Increasing trends in incidence of overweight and obesity over 5 decades. Amer J Med 2007; 120: 242-250.

3. YUSUF $S$, HAWKEN $S$, OUNPUU $S$, BAUTISTA L, FRANZOSI M, COMMEFORD P, et al. INTERHEART Study Investigators. Obesity and the risk of myocardial infarction in 27000 participants from 52 countries: a case-control study. Lancet 2005; 366: 1640-1649.

4. MINISTERIO DE SALUD. Encuesta Nacional de Salud, 2003. Minsal, Chile, http://epi.minsal.cl/epi/html/invest/ ENS/ENS.htm. (Última consulta al 30 de octubre de 2009).

5. MUSTA, SPADANO J, COAKLEY E, FIELD A, COLDITZ G, DIETZ W. The disease burden associated with overweight and obesity. JAMA 1999; 282: 1523-1529.

6. LANAS F, AVEZUM A, BAUTISTA L, DIAZ R, LUNA M, ISLAM S, et al. Risk factors for acute myocardial infarction in Latin America. The INTERHEART Latin America Study. Circulation 2007; 115: 1067-1074.

7. JORDAN J, ENGELI S, REDON J, SHARMA A, LUFT F, NARKIEVICZ K, et al. European Society of Hypertension Working Group on obesity: background, aims and perspectives. J Hypertens 2007; 25: 897-900.

8. FASCE E, CAMPOS I, IBAÑEZ P, FLORES M, ZÁRATE $\mathrm{H}, \mathrm{ROMÁN} \mathrm{O}$. Trends in prevalence, awareness, treatment and control of hypertension in urban communities in Chile. J Hypertens 2007; 25: 1807-1811.

9. KEARNEY P, WHELTON M, REYNOLDS K, MUNTNER P,
WHELTON P, HE J. Global burden of hypertension: analysis of worldwide data. Lancet 2005; 365: 217-223.

10. Encuesta de Caracterización Socioeconómica Nacional. Ministerio de Planificación, Chile. http://mideplan..cl/casen/ (último acceso el 29 de octubre 2009).

11. O'BRIEN E, ASMAR R, BEILIN L, IMAI Y, MALLION JM, MANCIA G, et al. European Society of Hypertension recommendations for conventional, ambulatory and home blood pressure measurement. J Hypertens 2003; 21: 821 848.

12. MINISTERIO DE SALUD. Guías del examen preventivo del adulto. Minsal, Chile 2000. http://minsal.cl/isi/guiasclinicas/ medicina_preventiva_ges.pedf (Último acceso el 29 de octubre 2009).

13. WOLF-MAIER K, COOPER R, KRAMER H, BANEGAS J, GIAMPAOLI S, JOFFRES M, et al. Hypertension treatment and control in five European countries, Canada and the United States. Hypertension 2004; 24: 10-17.

14.LAWES C, VANDER HOORN S, LAW M, ELLIOT P, McMAHON S, RODGERS A, et al. Blood pressure and the global burden of disease 2000. Part 1: Estimates of blood pressure levels. J Hypertens 2006; 24: 413-422.

15. PEREIRA $M$, LUNET $N$, AZEVEDO A, BARROS $\mathrm{H}$. Differences in prevalence, awareness, treatment and control between developing and developed countries. J Hypertens 2009; 27: 963-975.

16. UESHIMA H, ZHANG X, CHOUDHURY S. Epidemiology of hypertension in China and Japan. J. Hum Hypertens 2000; 14 765-769.

17.JADUE L, VEGA J, ESCOBAR M, DELGADO I, GARRIDO C, LASTRA P, el al. Factores de riesgo para las 
enfermedades no transmisibles: Metodología y resultados globales de la encuesta de base del programa CARMEN (Conjunto de Acciones para la Reducción Multifactorial de las Enfermedades No transmisibles. Rev Méd Chile 1999; 127: 729-738.

18. GUAGNANO M, BALLONE E, COLAGRANDE V, DELLA VECCHIA R, MANIGRASSO M, MERLITTI D, et al. Large waist circumference and risk of hypertension. Int $\mathrm{J}$ Obes Relat Metab Disord 2001; 25: 1360-1364.

19. KANAI H, MATSUZAWA Y, KITANI K, KENO Y, KOBATAKE T, NAGAI Y, et al. Close correlation of intra-abdominal fat accumulation to hypertension in obese women. Hypertension 1990; 16: 484-490.

20. SIANI A, CAPPUCCIO F, BARBA G, TREVISAN M, FARINARO E, LACONE R, et al. The relationship of waist circumference to blood pressure: the Olivetti Heart Study. Am J Hypertens 2002; 15: 780-786.

21.ZHU S, WANG Z, HESHKA S, HEO M, FAITH $M$, HEYMSFIELD $S$. Waist circumference and obesityassociated risk factors among whites in the third National Health and Nutrition Examination Survey: clinical action thresholds. Am J Clin Nutr 2002; 76: 743-749.

22. POIRIER P, LEMIEUX I, MAURIÉGE P, DEWAILLY E, BLANCHET C, BERGERON J, et al. Waist circumference on the relationship between blood pressure and insulin. Hypertension 2005; 45: 363-367.

23. HO S, CHEN Y, WOO J, LEUNG S, LAM T, JANUS E. Association between simple anthropometric indices and cardiovascular risk factors. Int J Obes Relat Metab Disord 2001; 25: 1689-1597.

24. HAYASHI T, BOYKO E, LEONETTI D, MACNEELY M, NEWELL-MORRIS L, KAHN S, et al. Visceral adiposity and the prevalence of hypertension in Japanese Americans. Circulation 2003; 108:1718-1723.

25.DING J, VISSER M, KRITCHEVSKY A, NEVITT $M$, SUTTON-TYRRELL K, HARRIS T. The association of regional fat depots with hypertension in older persons of white and African American ethnicity. Am J Hypertens 2004; 7 : 971-976.
26.DOLL S, PACCAUD F, BOVET P, BURNIER $M$, WIETLISBACH V. Body mass index, abdominal adiposity and blood pressure: consistency of their association across developing and developed countries. Int $\mathrm{J}$ Obes Relat Metab Disord 2002; 26: 48-57.

27.DYER A, LIU K, WALSH M, KIEFE C, JACOBS D, BILD D. Ten-year incidence of elevated blood pressure and its predictors: the CARDIA Study Coronary Artery Risk Development in (Young) Adults. J Hum Hypertens 1999; 13: $13-21$.

28. JUHAERI S, CHAMBLESS L, TYROLER H, ROSAMOND W, NIETO F, SCHREINER P, et al. Associations between weight gain and incident hypertension in a bi-ethnic cohort: the Atherosclerosis Risk in Communities Study. Int Obes Relat Metab Disord 2002; 26: 58-64.

29. OKOSUN I, CHANDRA K, CHOI S, CHRISTMAN J, DEBER G, PREWITT T. Hypertension and type 2 diabetes comorbidity in adults in the United States: risk of overall and regional adiposity. Obes Res 2001; 9: 1-9.

30. POIRIER P, DESPRES J. Waist circumference, visceral obesity, and cardiovascular risk. J. Cardiopulm Rehabil 2003; 23: 161-169.

31. THE OBESITY IN ASIA COLLABORATION. Is central obesity a better discriminator of the risk of hypertension than body mass index in ethnically diverse populations? J Hypertens 2008; 26: 169-177.

32. WEYER C, PRATLEY R, SNITKER S, SPRAUL $M$, RAVUSSIN E, TATARANNI P. Ethnic differences in insulinemia and sympathetic tones as links between obesity and blood pressure. Hypertension 2000; 36: 531-537.

33. MAJANE O, NORTON G, MASEKO M, MAKAULA S, CROWTHER N, PAIKER $\mathrm{J}$, et al. The association of waist circumference with ambulatory blood pressure is independent of alternative adiposity indices. J Hypertens 2007; 25: 1798-1806.

34.FASCE E. Hipertensión arterial y obesidad. SAIEH C, ZEHNDER C, editores. Hipertensión Arterial. Santiago de Chile, Mediterráneo 2008, 327-334. 